\title{
DAS STAATSANGEHÖRIGKEITSRECHT DER KOLONIEN IN AMERIKA
}

\author{
Von Hellmuth Hecker
}

Nachdem sowohl Nord- als auch Südamerika lange Zeit ausschließlich Kolonialgebiet europäischer Staaten gewesen waren, begann mit der Unabhängigkeitserklärung der USA von 1778 ein Prozeß der Verselbständigung des gesamten Kontinentes. Heute gibt es - nachdem Dänemark, Portugal, Schweden, Spanien als Kolonialmächte in diesem Raum ausgeschieden sind - nur noch Kolonien von drei europäischen Staaten, nämlich

1. vier französische Kolonien: Französisch-Guayana, Guadeloupe, Martinique (alle drei Übersee-Departements) und St. Pierre et Miquelon (Übersee-Territorium)

2. zwei niederländische Kolonien: Surinam (Niederländisch-Guayana) und Antillen (Curaçao)

3. vierzehn britische „Kolonien“, wovon sechs assoziierte Staaten sind.

Außerdem besitzen die USA noch drei abhängige „Kolonialgebiete“, nämlich: Jungfern-Inseln, Panama-Kanalzone, Puerto Rico.

\section{AMERIKANISCHE KOLONIEN}

Unter dem Ausdruck „Kolonien“ sind hier drei Gebiete des amerikanischen Erdteils zu verstehen, die nicht wie etwa Alaska Gliedstaat der USA sind. Diese Gebiete wurden erworben:

1. durch Kauf, die Jungfern-Inseln 1916 von Dänemark

2. durch Verwaltungszession, die Panama-Kanalzone 1903 von Panama

3. durch Eroberung, Puerto Rico 1898 von Spanien

In allen drei Gebieten gilt das StA-Recht der USA nicht direkt

1. JUNGFERN-INSELN

St. Croix und St. John waren zuerst britisch, seit 1667 dänisch. Frankreich trat 1733 St. Thomas an Dänemark ab. 1916/17 erwarben die USA die drei Inseln.

4. 8. 1916 Dänisch-amerikanischer Vertrag über den Erwerb der Virgin Islands StAB: Art. 6

in Kraft: 17.1.1917

Amtlicher Text: Statutes Bd. 39, S. 171; TS 629

Englischer Text: Flournoy, S. 652 (Art. 6)

Virgin Islands code, Washington 1957, Bd. 1, S. XLV

State Papers Bd. 110, S. 843

Martens, N. R. G., 3. Serie, Bd. 10, S. 357 
25. 2. 1927 Gesetz betr. St A und Einbürgerung für die Virgin Islands, Nr. 640

5 Sections

Amtlicher Text: Statutes Bd. 44, S. 1234

Englischer Text: Flournoy, S. 625

Hackworth, Bd. 3, S. 150

State Papers Bd. 127, S. 940

Lewis, 1953, S. 15

Französische Ưbersetzung: Bourbousson, S. 358

28. 6. 1932 Gesetz betr. Einwanderung und Einbürgerung gewisser Eingeborener der Virgin Islands, Nr. 198

StAB: Sect. 5 ändert Sect. 1 v. 1927

Englischer Text: Lewis, 1953, S. 37

27. 6. 1952 StAG der USA

StAB für Virgin Islands: Sect. 306

Englischer Text: LCN, S. 498

\section{PANAMA-KANALZONE}

Die Zone wurde durch Vertrag v. 18.11.1903 von Panama zur ständigen Verwaltung erworben.

4. 8. 1937 Gesetz betr. St A in der Kanalzone, Nr. 242, c. 563

Amtlicher Text: Statutes Bd. 50, S. 558

Englischer Text: Lewis, 1953, S. 60

14. 10. 1940 StAG der USA

StAB f. Kanalzone: Sect. 203. Nach Sect. 101 (e) war die Kanalzone keine „outlying possession “, für die besondere StABen galten

27. 6. 1952 StAG der USA

StAB f. Kanalzone: Sect. 303 (entspricht Sect. 203 v. 1940)

\section{PUERTO RICO}

Portorico 1898 von Spanien an die USA abgetreten, StAB im Friedensvertrag von 1898 Art. 9 für alle abgetretenen Gebiete (s. Cuba). Seit 1932 amtlich Puerto Rico.

12. 4. 1900 Gesetz über die Regierung von Portorico, c. 191

In Kraft: 1. 5. 1900

StAB: Sect. 7

Amtlicher Text: Statutes Bd. 31, S. 77

Englischer Text: State Papers Bd. 92, S. 742

Flournoy, S. 623 (Art. 7)

2. 3. 1917 Gesetz über die Regierung von Portorico, c. 145

StAB: Sect. 5 und 41

Amtlicher Text: Statutes Bd. 39, S. 951

Englischer Text: State Papers Bd. 112, S. 1169

Flournoy, S. 623 (Sect. 5 und 41)

Französische Übersetzung: Bourbousson, S. 359

4. 3. 1927 Gesetz zur Änderung des Gesetzes v. 2. 3. 1917, c. 503

StAB: Sect. 2 fügt neue Sect. 5 A ein

Amtlicher Text: Statutes Bd. 44, S. 1418

Englischer Text: Flournoy, S. 624 (Sect. 5a) 
27. 6. 1934 Gesetz zur Änderung des Gesetzes v. 2. 3. 1917, Nr. 477, c. 845

StAB: Sect. 5 B eingefügt

Amtlicher Text: Statutes Bd. 48, S. 1245

Englischer Text: State Papers Bd. 137, S. 703

Lewis, 1953, S. 44

26. 3. 1938 Gesetz zur Änderung des Gesetzes v. 2. 3. 1917, c. 51

StAB: Sect. 41 geändert

Amtlicher Text: Statutes Bd. 52, S. 118

16. 5. 1938 Gesetz zur Änderung des Gesetzes v. 2. 3. 1917, Nr. 521, c. 225

StAB: Sect. $5 \mathrm{C}$ eingefügt

Amtlicher Text: Statutes Bd. 52, S. 377

Englischer Test: Lewis, 1953, S. 64

14. 10. 1940 StAG der USA

In Kraft: 13.1.1941

StAB f. Puerto Rico: Sect. 202 (entspricht Sect. 5 C des Gesetzes von 1938) und Sect. 322

Amtlicher Text: Statutes Bd. 54, S. 1139

25. 6. 1948 Gesetz zur Änderung des Gesetzes v. 2. 3. 1917, Nr. 776, c. 649

StAB: Sect. 5 B geändert

Amtlicher Text: Statutes Bd. 62, S. 1015

27. 6. 1952 StAG der USA

StAB f. Puerto Rico: Sect. 302 (entspricht Sect. 202 des Gesetzes von 1940)

Literatur:

Crone, L. E.

Citizenship status of inhabitans of the territories and outlying possessions, in: Imm. and natural. service, 2. Serie, Nr. 35 v. 11. 2. 1935 S. 6-8 (Puerto Rico) und Nr. 36 v. 18. 2. 1935, S. 4-6 (Jungferninseln), 6-8 (Kanalzone)

Capó-Rodríguez, P. Relations between the United States and Porto Rico, in: AJIL 1919, S. 483 ff. (S. 504-511: American and Porto Rican citizenship)

Falkner, R. P.

Hadkworth, G. H.

Citizenship for the Porto Ricans, in: APSR 1910, S. $180-195$

Digest of international law, Bd 3, Washington 1942, S. 117-119 (Kanalzone), 147-154 (Jungfern-Inseln), S. 141-147 (Puerto Rico).

Mc Govney, D. O.

o. V.

Our non-citizen nationals, who are they?

in: California Law Review 1933/4 (Bd. 22), S. 593-635 (insb. auch Puerto Rico)

Political status of Porto Ricans,

in: Harvard Law Review 1903/4 (Bd. 17), S. 412-413

Allgemein zur StA bei Erwerb der spanischen Kolonien

Morse, A. P.

Pingrey, D. $\mathrm{H}$.

Civil and political status of inhabitants of ceded territories, in: Harvard Law Review 1900/1 (Bd. 14), S. 262-272

Rights of inhabitans of acquired territory, in: Chicago Legal News 1898 (Bd. 31), S. $32 \mathrm{ff}$.

Stillmann, J. W.

Citizenship in ceded territory in: Green Bag (Boston) 1899 (Bd. 11), S. $203 \mathrm{ff}$.

o. V.

Citizenship in our new possessions,

in: Albany Law Journal 1899 (Bd. 59), S. $247 \mathrm{ff}$ 


\section{BRITISCHE KOLONIEN}

Vom britischen Herrschaftsgebiet in Amerika schieden durch Unabhängigkeit folgende Gebiete im Laufe der Zeit aus:

Vereinigte Staaten: 4. 7.1778

Canada:

seit 1931 Dominion

Jamaica:

7. 8.1962

Trinidad/Tobago:

30. 8.1962

Guayana:

26. 5.1966

Barbados:

30. 11.1966

Eine Übersicht über das Staatsangehörigkeitsrecht der letztgenannten vier Staaten ist zu finden in: StAZ 1968, S. 25-26. Die USA sind behandelt in Bd. 7 und 7 a der SGS. Canada ist bisher noch nicht erfaßt worden.

Die bei Großbritannien verbliebenen Gebiete umfassen 14 Territorien, von denen sechs als assoziierte Staaten eine eigene Staatsangehörigkeit besitzen können (Antigua, Dominica, Grenada, St. Kitt's, St. Lucia und St. Vincent), während die übrigen acht Kolonien sind. Sie haben meist diverse Vorschriften über lokale Einbürgerung und örtliche Anwendung des britischen Staatsangehörigkeitsrechts erlassen. Der britische West Indies Act v. 1967 (c. 4), der die Gründung der Assoziierten Staaten vorsah, bestimmte in Sect 12, wie die StA von deren Bürgern zu bezeichnen sei.

\section{ANTIGUA}

Seit 1666 endgültig britisch, 1860 wurde Barbuda angegliedert, assoziierter Staat ab 27. 2. 1967

Okt. 1861 Gesetz Nr. 739 betr. Einbürgerung von Neger-Einwanderern

(siehe Foreign Relations 1873 II, S. 1255)

22. 2. 1967 Antigua Constitution Order in Council

StAB: Sect. 94-101

Amtlicher Text: S. I. 1967, Nr. 225

\section{BAHAMAS}

Seit 1718 britische Kronkolonie, 1781-83 spanisch, 1848 Abtretung der Turks und Caicos Inseln an Jamaica

22. 3. 1848 Alien Act, Nr. 4 (betreffend Einbürgerung)

(2 Vict. c. 4)

Dies Gesetz galt bis 1968 auf den Turks und Caicos Inseln weiter

Amtlicher Text: Statute Law of the Bahama Islands 1799-1929, c. 257 , S. 1929

Englischer Text: State Papers Bd. 68, S. 1110

11. 5. 1866 Alien Amendment Act, Nr. 15

(29 Vict. c. 15)

Englischer Text: State Papers Bd. 68, S. 1113

11. 4. 1872 Alien Amendment Act, Nr. 9

(35 Vict. c. 9)

Englischer Text: State Papers Bd. 65, S. 1221 
23. 5. 1872 Naturalization Act, Nr. 20

(35 Vict. c. 20)

Englischer Text: State Papers Bd. 65, S. 1222

1916 Alien Amendment Act, Nr. 5

1919 Alien Amendment Act, Nr. 10

1928 Alien Amendment Act, Nr. 3

1. 8. 1950 Status of Aliens Act, Nr. 11

Enthält keine StAB, sondern regelt nur Landerwerb durch Ausländer

Amtlicher Text: Rev. Ed., c. 180

Rev. Ed. 1965, c. 237

\section{BERMUDA}

Seit 1684 britische Kronkolonie

1857 Aliens Act, Nr. 11

2. 10. 1871 Naturalization Act (Aliens Property)

Englischer Text: State Papers Bd. 65, S. 1303

21. 10. 1897 Aliens Act,

Englischer Text: State Papers Bd. 90, S. 937

1. 3. 1900 Aliens Amendment Act

15. 7.1901 Aliens Act,

Englischer Text: State Papers Bd. 95, S. 203

20. 3.1907 Alien Act, Nr. 12 (betr. Einbürgerung)

Aufgehoben durch Alien Act Nr. 18 von 1926 (i. K. 1. 5. 1926), der keine StAB mehr enthält

Englischer Text: State Papers Bd. 100, S. 620

18. 1. 1911 Alien Amendment Act, Nr. 3

Englischer Text: State Papers Bd. 104, S. 321

7. 7.1915 Naturalization Act, Nr. 18

17. 3. 1921 Aliens Amendment Act, Nr. 19

Englischer Text: State Papers Bd. 116, S. 283

17. 2. 1928 Aliens Amendment Act, Nr. 12, betr. Verlust der Einbürgerung

Englischer Text: State Papers Bd. 128, S. 178

20. 6. 1949 Brit. Naturalization (Supp. Prov.) Act, Nr. 46

Bereinigt durch Statutory Law Rev. Act Nr. 78 von 1951

Amtlicher Text: Statute Law of the Bermudas 1620-1952, c. 25, S. 1198

Laws, Rev. Ed. 1964, Title 5 (A)

1950 Brit. Naturalization Amendment Act, Nr. 57

1952 Brit. Naturalization Amendment Act, Nr. 33

12. 7. 1962 Brit. Naturalization Amendment Act, Nr. 100

Amtlicher Text: Acts 1962, S. 495 (neue Schedule)

\section{BRIT. ANTARCTIS TERRITORY}

siehe Falkland-Inseln

\section{BRITISH HONDURAS}

Erste britische Siedlung an der Mosquito-Küste 1630, 1739 britisch, 1763 Verzicht auf Rechte, 1786 endgültig Kronkolonie, 1862-1884 Jamaica unterstellt, 1852-1859 bildeten die Ruatan-Inseln eine eigene Kronkolonie 
19. 7. 1855 Naturalization of Aliens Act, c. 18

Englischer Text: State Papers Bd. 69, S. 316

1861 Immigration Act, c. 5

StAB: Sect. 23

1883 Naturalization of Aliens Ordinance

Amtlicher Text: Rev. Ed. 1887, Bd. 4, c. 38

Englischer Text: State Papers Bd. 82, S. 1293

1885 Naturalization of Aliens (Amd) Ordinance

Englischer Text: State Papers Bd. 82, S. 1298

1914 Naturalization Ordinance, Nr. 56

Amtlicher Text: Consolidated Laws of British Honduras 1924, c. 114, S. 680

1915 British Nationality (Fees) Ordinance, Nr. 32

28. 3. 1928 Naturalization Amendment Ordinance Nr. 12

1. 10. 1949 British Nationality Act 1948 (Local Provisions) Ordinance, Nr. 13 Amtlicher Text: Consolidated Laws of British Honduras 1953, c. 110 Laws of British Honduras, Rev. Ed. 1957, c. 107, S. 1736

\section{CAYMAN INSELN}

Seit 1660 britisch besiedelt, 1863 Jamaica unterstellt, seit 20. 2. 1958 eigene Kolonie. Staatsangehörigkeitsvorschriften waren nicht feststellbar.

\section{DOMINICA}

Seit 1763 endgültig britisch, 1764-1771 zu Windward-Inseln, 1778-1783 wieder französisch, 1833-1939 zu Leeward-Inseln, seitdem Kolonie, innerhalb der Windward-Inseln, seit 1. 3. 1967 assoziierter Staat.

1950 Dominica (Deprivation of Citizenship) Rules

Amtlicher Text: S. R. O. Nr. 23

22. 2. 1967 Dominica Constitution Order in Council

i. K.: 1. 3. 1967

StAB: Sect. 88-95

Amtlicher Text: S. I. 1967, Nr. 226

\section{FALKLAND INSELN}

Nach wechselndem Geschick 1832 britisch, 1843 Kronkolonie, 1908 Teile der Antarctis als Dependenz, diese seit 3. 3. 1962 als Brit. Antarctis Territory eigene Kolonie (außer Südgeorgien und Sandwich-Inseln). Argentinien beansprucht die Bewohner als eigene Staatsangehörge.

1891 Naturalization Ordinance, Nr. 2

14. 12. 1891 Naturalization Amendment Ordinance, Nr. 9

Englischer Text: State Papers Bd. 85, S. 138

27. 7.1900 Alien Ordinance Nr. 5, betr. Einbürgerung

Amtlicher Text: Laws, Rev. Ed. 1915, S. 197

Englischer Text: State Papers Bd. 94, S. 123

1928 Alien (Amendment) Ordinance

3. 5. 1929 Local Naturalization Ordinance Nr. 4

Ersetzt die von 1900 und 1928

Englischer Text: State Papers Bd. 130, S. 87

31. 12. 1949 Brit. Nationality Ordinance, Nr. 25

Amtlicher Text: Laws, Rev. Ed. 1951, c. 6, Bd. 1, S. 39 


\section{GRENADA}

Seit 1650 französisch, seit 1762 britisch (außer 1779-1783), als Kolonie der Windward-Inseln, seit 3. 3. 1967 assoziierter Staat.

1858 Gesetz Nr. 230 betr. Einbürgerung von Neger-Einwanderern

7. 10. 1872 Naturalization of Aliens Act, Nr. 118

aufgehoben (erledigt) durch Ordinance Nr. 6 von 1949

Amtlicher Text: Statutes, Rev. Ed. 1911, c. 139

Laws, Rev. Ed. 1934, c. 145, S. 1535

Englischer Text: State Papers Bd. 65, S. 1286

26. 7. 1915 Brit. Naturalization and Status of Aliens Ordinance, Nr. 13

Amtlicher Text: Laws, Rev. Ed. 1934, c. 25, S. 165

22. 2. 1967 Grenada Constitution Order in Council

StAB: Sect. 95-102

Amtlicher Text: S. I. 1967, Nr. 227

\section{LEEWARD-INSELN (MONTSERRAT UND VIRGIN ISLANDS)}

Seit 1625 britische Rechte auf die Inselgruppe, 1672 von Barbados getrennte Kolonie. 1871 Föderation aus sechs Inseln (Antigua, Dominica, Montserrat, Nevis, St. Kitt's, Virgin Islands), 1882 Nevis und St. Kitt's vereinigt, 1939 Dominica an Windward-Inseln angegliedert, 1967 Antigua und St. Kitt's-Nevis als assoziierte Staaten abgetrennt. Die Kolonie besteht seitdem aus nur zwei Präsidentschaften: Montserrat (seit 1632 britisch) und die britischen Jungfern-Inseln (seit 1666).

29. 7. 1880 Aliens Act, Amendment Law, Nr. 4

Amtlicher Text: Rev. Ed. 1914

Englischer Text: State Papers Bd. 71, S. 296

7. 3. 1916 Naturalization Documents and Fees Act, Nr. 11

Amtlicher Text: Federal Acts of the Leeward Islands, Rev. Ed. 1927, c. 78

1921 Naturalization Amendment Act, Nr. 14

4. 5. 1928 Naturalization Law Amendment Act, Nr. 3

hebt auf u. a. das Gesetz von 1880

\section{ST. CHRISTOPHER (KIT'T'S), NEVIS und ANGUILLA}

Die drei Inseln St. Kitt's (St. Christopher), Nevis und Anguilla waren seit 1625 als Teile der Leewardinseln britisch. St. Kitt's war im 17./18. Jahrhundert zeitweise französisch. Anguilla wurde 1883 mit St. Kitt's vereinigt. Am 27. 2. 1967 wurden die drei Inseln assoziierter Staat. Am 30. 5. 1967 erklärte sich Anguilla einseitig für unabhängig, was aber von niemandem anerkannt wurde.

3. 2. 1857 Aliens Act insb. betr. befreite Sklaven

Amtlicher Text: The Statutes of The Islands of St. Christopher and Anguilla, Rev. Ed. 1857, Nr. 127, S. 368

22. 2. 1967 Constitution Order in Council

i. K.: 27. 2. 1967

StAB: Sect. 90-97

Amtlicher Text: S. I. 1967, Nr. 228 


\section{ST. LUCIA}

Nach Wechsel der Herrschaft zwischen England und Frankreich ist die Insel seit 1815 endgültig britisch, seit 1838 Kolonie innerhalb der Windward-Inseln. Seit

1. 3.1967 assoziierter Staat.

11. 4. 1857 Aliens Act

20. 9. 1901 Naturalization Ordinance, Nr. 6

Englischer Text: State Papers Bd. 95, S. 338

23. 10. 1915 Brit. Nat. and Status of Aliens (Fees) Ordinance, Nr. 17

Amtlicher Text: Rev. Ed. 1916, Nr. 126, S. 940

22. 2. 1967 St. Lucia Constitution Order in Council

StAB: Sect. 88-95

Amtlicher Text: S. I. 1967, Nr. 229

\section{ST. VINCENT}

Nach Wechsel der Herrschaft zwischen England und Frankreich seit 1783 endgültig britisch. Seit 1833 als Kolonie unter den Windward-Inseln. Am 29. 5. 1967 wurde die geplante Staatlichkeit verschoben und trat erst am 27. 10. 1969 in Kraft.

Okt. 1857 Gesetz über Einwanderung

StAB: Sect. 17 (Neger-Einbürgerung)

27. 3. 1902 Naturalization Ordinance, Nr. 8

Amtlicher Text: Rev. Ed. 1911

Englischer Text: State Papers Bd. 95, S. 340

6. 7.1905 Naturalization Amendmend Ordinance

Englischer Text: State Papers Bd. 98, S. 611

15. 12. 1915 Brit. Nationality and Status of Aliens Ordinance, Nr. 26

Amtlicher Text: Rev. Ed., c. 39, S. 465

Englischer Text: State Papers Bd. 109, S. 627

1950 Brit. Nationality Ordinance, Nr. 13

1951 Deppatation (Brit. Subjects) Ordinance, Nr. 30

26. 5. 1954 Naturalization Amendment Ordinance, Nr. 19

Amtlicher Text: Ordinances 1954, S. 33

9. 5. 1956 Naturalization Amendment Ordinance, Nr. 20

Amtlicher Text: Ordinances 1956, S. 99

22. 10. 1969 St. Vincent Constitution Order

StAB: S. 91-98

Amtlicher Text: S. I. Nr. 1500

\section{TURKS AND CAICOS ISLANDS}

Seit 1670 britisch besiedelt, 1799 Kolonie unter den Bahamas. 1848-1873 eigene Kolonie, 1873-1958 unter Jamaica.

Seit 20. 2. 1958 von Jamaica abgetrennte Kolonie

22. 3. 1848 Gesetz Nr. 4 betr. Einbürgerung von Ausländern

Nr. 11, Vict. c. 4

Dies Gesetz war der Aliens Act der Bahama Inseln (s. o.) und wurde nach der Abtrennung der Turks und Caicos Inseln dort in Kraft gelassen. Erst durch die Ordinance Nr. 5, in Kraft 27. 7. 1968, wurde das Gesetz in der neuen Rev. Ed. gestrichen.

Amtlicher Text: siehe oben Bahamas

Englischer Text: siehe oben Bahamas 


\section{FRANZOOSISCHE KOLONIEN}

Nachdem Frankreich, das 1763 Kanada verloren hatte, seine letzten Festlandsbesitzungen (Louisiana) 1803 an die USA abgetreten hatte, blieben ihm ab 1814 nur noch einige Inseln sowie auf dem südamerikanischen Kontinent Französisch Guayana. Der Inselbesitz besteht aus St. Pierre et Miquelon (Rest des Besitzes von Kanada) im Norden sowie den Antillen Guadeloupe und Martinique (Rest des Besitzes von Haiti) im Süden. Die Antillen und Guayana sind seit Gesetz v. 19. 3. 1946 Überseedepartements, St. Pierre et Miquelon ist Überseeterritorium. Alle Bewohner besitzen die volle französische StA ab dem Gesetz v. 7. 5. 1946, das allen Kolonien die StA gewährte.

Literatur:

Cogordan

S. 396-398 (betr. St. Barthelemy)

Thamar

Le "Nègre créole" martiniquais,

in: Revue juridique et économique du Sud-Ouest 1965, S. 47-72

\section{FRANZOSISCH GUAYANA}

Seit 1604 französisch, außer 1654-77 (brit.) und 1809-17 (portugiesisch). Die französischen StAG'e galten nicht unmittelbar für Guayana. Erst 1946 wurde der StA-Code von 1945 auf Guayana erstreckt.

27. 9. 1946 Dekret Nr. 2094 betr. Erstreckung des StA-Code auf Guayana

Amtlicher Text: J. O. v. 29. 9. 46, S. 8288

Französischer Text: Recueil 1965, S. 4

31. 3. 1955 Gesetz Nr. 357 betr. Änderung v. Art. 11 der Mantelordonnanz zum StA-Code von 1945

Art. 11 wurden zwei weitere Absätze angefügt, die Sonderregeln für den StA-Erwerb der Bewohner von Guayana enthalten

Amtlicher Text: J. O. v. 1. 4. 1955, S. 3223

Deutsche Übersetzung: StAZ 1957, S. 22

\section{GUADELOUPE UND MARTINIQUE}

Beide Inseln sind ab 1653 französisch, endgültig von Großbritannien anerkannt 1815. Seit dem Senatskonsult vom 3. 5. 1854 unterliegen sie als "alte Kolonien“ zusammen mit Réunion einer besonderen und gemeinsamen Regelung. Die StAGesetze von 1889 (Art. 2), 1927 (Art. 15 I) und 1945 (Art. 11 der Ordonnance) galten auch für sie.

Guadeloupe hat drei Dependencien: Desiderade (französisch seit 1728), St. Martin Nordteil (französisch seit 1639, der Süden ist holländisch), Barthélemy (französisch ab 1648, 1784-1877 schwedisch).

2. 3. 1878 Gesetz betr. Billigung v. Ausführung des französisch-schwedischen Protokolls über die StA der Bewohner von St. Barthélemy

v. 31. 10. 1877

Inkrafttreten des Vertrages .v. 10. 8. 1877, dessen Bestandteil das Protokoll bildet, am 6. 3. 1878

StAB: Art. 1-2

Das Vertragswerk wurde durch Gesetz v. 2. 3. 1878 (J. O. v. 3. 3.) gebilligt und ausgeführt, durch Dekret v. 12. 3. 1878 veröffentlicht. 
Durch Dekret v. 27. 7. 1878 wurde die Insel an Guadeloupe angegliedert.

Texte des Vertragswerkes:

Amtlicher Text: J. O. v. 13. 3. 1878

Französischer Text: CNF Bd. 2, S. 994

Martens, N. R. G., 2. Serie, Bd. 4, S. 367

6. 10. 1939 Dekret betr. Ausdehnung des Teils III des Dekrets v. 12. 11. 1938 auf die drei alten Kolonien

Das Dekret von 1938 hatte das StAG von 1927 geändert, enthielt aber keine Erstreckung auf die Antillen

Amtlicher Text: J. O. v. 10. 10. 39, S. 12223

Französischer Text: CNF Bd. 1, S. 136

11.11. 1939 Dekret betr. Ausdehnung des ersten Dekrets v. 19. 10. 1939 auf die drei alten Kolonien

Das Dekret v. 19. 10. 39 hatte Art. 14 (a) des StAG von 1927 geändert.

Amtlicher Text: J. O. v. 14. 11. 1939, S. 13109

Französischer Text: CNF Bd. 1, S. 137

11. 5. 1940 Dekret betr. Ausdehnung des Dekrets v. 9. 3. 1940 auf die alten Kolonien

Das Dekret hatte Art. 9 Nr. 1 II des StAG von 1927 geändert

Amtlicher Text: J. O. v. 19. 5. 1940, S. 3713

Französischer Text: CNF Bd. 1, S. 202

\section{ST. PIERRE ET MIQUELON}

Die Inseln sind seit 1635 französische Kolonie, seit 1946 Überseeterritorium. Die Bewohner besitzen seit 1946 die volle französische StA. Besondere Vorschriften für die Inseln sind im StA-Recht nicht ergangen.

\section{NIEDERLANDISCHE KOLONIEN}

Die Bewohner der beiden niederländischen Kolonien (Surinam und Antillen) waren zunächst reine Schutzgenossen, ab 1927 koloniale Untertanen, seit Gesetz v. 21. 12. 1951 gleichberechtigte Niederländer. Der Begriff „Königreich“ umfaßt seither auch die beiden Kolonien.

\section{ANTILLEN (CURACAO)}

Die niederländischen Antillen sind endgültig seit 1648 holländisch und hießen bis 1950 Curaçao. Für die StA gilt dasselbe wie für Surinam.

4. $9.1868 \mathrm{Kgl}$. Beschluß Nr. 18 betr. Übergangsgesetzgebung in Curaçao

i. K.: 1. 5. 1869

StAB: Art. 10

Amtlicher Text: Publicatieblad Nr. 16

1927-1969 siehe Surinam

\section{SURINAM (NIEDERLANDISCH GUAYANA)}

Der mittlere Teil von Guayana wurde 1667 von den Niederlanden im Tausch gegen New York von England erworben und erhielt den Namen Surinam. Die Bewohner erhielten durch das Gesetz v. 10. 6. 1927 die Stellung von holländischen 
„Untertanen“ im Gegensatz zu mutterländischen Niederländern. Heute sind die Bewohner von Surinam niederländische StA'e: eine Landesangehörigkeit kann örtlich geregelt werden (Art. 6 der Staatsregeling von 1955).

4. 9. $1868 \mathrm{Kgl}$. Beschluß Nr. 17 betr. Übergangsgesetzgebung in der Kolonie Surinam

i. K.: 1. 5. 69

StAB: Art. 10

Amtlicher Text: GvBl. Nr. 14

10. 6. 1.927 Gesetz betr. Ausdehnung des Gesetzes v. 10. 2. 1910 betr. Untertanenschaft in Niederländisch Indien auf Surinam und Curaçao

i. K.: 25. 7. 1927

Amtlicher Text: StBl. Nr. 175

Ind. St. Nr. 418

GvBl. Nr. 60

PBL Nr. 43

10. 5. 1929 und 21. 12. 1936

Gesetze betr. Änderungen des Gesetzes von 1910

Näheres s. Indonesien

Amtlicher Text: StBl. Nr. 258 (1929) und 912 (1936)

28. 12. 1951 Kgl. Beschluß, wonach in besonderen Fällen kein Verlust durch Auslandsdienst nach Art. 2 I Z. 3 des Gesetzes von 1910 erfolgt

Amtlicher Text: StBl. Nr. 603

3. 11. 1954 Gesetz betr. kostenlose Wiederverleihung der Niederländerschaft an in Surinam wohnende Indonesier

i. K.: 8. 11. 1954 (s. StBl. Nr. 477)

Amtlicher Text: StBl. Nr. 476

Holländischer Text: Practicus 3. 11

14. 9. 1962 Reichsgesetz betr. Aufhebung des Gesetzes von 1910, mit Übergangsregeln

Amtlicher Text: StBl. Nr. 358

PBl. Nr. 136

Holländischer Text: Practicus 2.4

14. 11. 1963 Reichsgesetz betr. Änderung von Art. 3 des Gesetzes vom 3. 11. 1954 Amtlicher Text: StBl. Nr. 467

Ismael, J.

De positie van de Indonesier in het nieuw Suriname,

De positie van de Indonesier in het nieu
in: Indonesie (Den Haag) 1954, Nr. $3 / 4$ 